\title{
Equivalence of Boltzmann and moment equations
}

\author{
R. Cubarsi \\ Dept. Matemàtica Aplicada IV, Universitat Politècnica de Catalunya, 08034 Barcelona, Catalonia, Spain \\ e-mail: rcubarsi@ma4 .upc . edu
}

Received 11 April 2010 / Accepted 30 June 2010

ABSTRACT

\begin{abstract}
The closure problem for the stellar hydrodynamic equations is studied by describing the family of phase space density functions, for which the collisionless Boltzmann equation is strictly equivalent to a finite subset of moment equations. It is proven that the redundancy of the higher-order moment equations and the recurrence of the velocity moments are of similar nature. The method is based on the use of maximum entropy distributions, which are afterwards generalised to phase space density functions depending on any isolating integral of motion in terms of a polynomial function of degree $n$ in the velocities. The equivalence between the moment equations up to an order $n+1$ and the collisionless Boltzmann equation is proven. It is then possible to associate the complexity of a stellar system, i.e., the minimum set of velocity moments needed to describe its main kinematic features, with the number of moment equations required to model it.
\end{abstract}

Key words. stars: kinematics and dynamics - Galaxy: kinematics and dynamics - galaxies: statistics - methods: analytical hydrodynamics

\section{Introduction}

A maximum entropy density function was used in Cubarsi (2010, hereafter Paper I), to infer the stellar velocity distribution from a finite set of moment constraints. An explicit relationship between higher-order moments and the moment constraints was also established. Now, as a corollary of the method, maximum entropy distributions allow us to formulate the equivalence of the collisionless Boltzmann equation and the stellar hydrodynamic equations. This result could be of interest in modelling the dynamics of some stellar groups and may be useful as well to astronomy students for clarifying some basic concepts of stellar dynamics.

The most basic cases of dealing with the stellar hydrodynamic equations are the continuity equation, for order $n=0$, standing for mass conservation, and the momentum conservation equation, for $n=1$, also called Jeans equation. It is well known that the infinite hierarchy of the stellar hydrodynamic equations cannot be used as a dynamical model to study a stellar system unless they are reduced to a finite number of equations and unknowns. In most applications, only the lowest order equations are used, in addition to some particular assumptions to restrict the number of unknowns; for example, by assuming the epicyclic approach (Oort 1965), axial symmetry (Vandervoort 1975), or by taking a velocity distribution function depending on specific integrals of the star motion (Jarvis \& Freeman 1985). In this way, some constraints between central moments are reached (van der Marel 1991). Obviously, when working with the Jeans equation alone, or with a finite set of hydrodynamic equations, the collisionless Boltzmann equation is not generally fulfilled. Then arises the closure problem of how the infinite hierarchy of moment equations and unknowns are reduced to a finite subset, which is still equivalent to the collisionless Boltzmann equation. It is worth mentioning the explicit treatment of the closure problem by Cuddeford \& Amendt (1991). They adopted some closure assumptions involving the moments of the velocity distribution, which did match some known constraints between the moments of the Schwarzschild distribution, like those related to the skewness and the kurtosis of the distribution in specific directions. In this way, they made the stellar hydrodynamic equations equivalent to the collisionless Boltzmann equation, as well as obtaining a phase-space density function which was more general than the Schwarzschild type. However, the closure conditions they found, even using up to eighth-order moments, were only valid in a steady-state, cool, axisymmetric stellar system, with vanishing radial mean velocity. Most of those assumptions are already non-valid in the solar neighbourhood.

In Cubarsi (2007), hereafter Paper II, the general form of the $n$-order stellar hydrodynamic equation was derived in terms of the comoving velocity moments in a completely analytical way and without any additional hypotheses. It was a previous step to study the closure problem, trying to answer the following questions: when is a finite subset of hydrodynamic equations strictly equivalent to the collisionless Boltzmann equation? Which are the redundant moment equations? Can we explicitly write the conditions that make them redundant? In other words, under which assumptions is it possible to find some general closure conditions? The answers to these questions may vary depending on the form of the velocity distribution. A first approach to the closure problem was discussed and solved for ellipsoidal velocity distributions. It was proven for a Schwarzschild distribution that the whole set of hydrodynamic equations was reduced to the moment equations of the orders $n=0,1,2,3$, which were equivalent to the collisionless Boltzmann equation. In a more general case, for an arbitrary ellipsoidal trivariate velocity distribution, some analogous closure conditions were obtained. In both cases, the closure conditions were provided by some recurrent relationships of the velocity moments, so that moments of fourth and higher orders were determined in terms of the lower-order moments. Therefore, it was suggested that the closure problem was related to the macroscopic properties of the velocity distribution, although different velocity distributions 
could have analogous closure conditions and a similar set of independent moment equations.

In the current paper, the closure problem is studied in a more general way, by describing the whole family of phase space density functions, for which the collisionless Boltzmann equation is strictly equivalent to a finite subset of hydrodynamic equations. In addition, it is proven that the redundancy of the higherorder moment equations and the recurrence of the velocity moments are of similar nature. The method is based on the use of maximum entropy distributions, which allow an easy algebraic treatment. The closure problem is solved in two steps. First, a family of maximum entropy distributions is considered, which is a generalisation of an exponential quadratic function to an exponential polynomial function of degree $n$ in the velocity variables. Second, the maximum entropy functions are taken as a basis of square-integrable functions, to expand any arbitrary, non-exponential function in terms of a $n$-degree polynomial, as a convergent power series. For such a general family of functions, i.e., the phase space density functions depending on any isolating integral of motion expressed as polynomial function in the velocities, the equivalence between the first set of moment equations, up to order $n+1$, and the collisionless Boltzmann equation is proven.

Furthermore, it is possible to associate the complexity of a stellar system, i.e., the minimum set of velocity moments needed to describe its main kinematic features, with the number of moment equations required to model it, as shown from samples used in the application section of Paper I, drawn from the HIPPARCOS and Geneva-Copenhagen survey catalogues.

The paper is organised as follows. In Sect. 2, the notation is introduced while reviewing the basic concepts of stellar dynamics to be used: the Boltzmann equation, a property of the isolating integrals of motion, and the moment equations. In Sect. 3, a maximum entropy density function is used to obtain the fundamental system of equations, associated with the collisionless Boltzmann equation, and the closure problem is solved for this family of functions. In Sect. 4, the closure problem is generalised to arbitrary phase density functions. Finally, some conclusions are pointed out.

\section{Basic concepts}

\subsection{Boltzmann equation}

It is generally assumed that the Galaxy is at present in a state in which each star can be idealised as a conservative dynamical system to a very high degree of accuracy. In general, the forces acting in the system can be associated with a gravitational potential function per unit mass $\mathcal{U}(t, r)$, so that the motion of a star is described in a cartesian coordinates system by the Hamiltonian system of equations

$\dot{\boldsymbol{r}}=\boldsymbol{V}, \quad \dot{\boldsymbol{V}}=-\mathcal{U}(t, \boldsymbol{r})$.

For the whole stellar system, the collisionless Boltzmann equation is satisfied (see Hénon 1982, for the appropriate terminology), so that the phase space density function $f(t, r, \boldsymbol{V})$, with $(t, \boldsymbol{r}, \boldsymbol{V}) \in R \times \Gamma_{r} \times \Gamma_{V}$, fulfils

$\frac{\mathrm{D} f}{\mathrm{D} t} \equiv \frac{\partial f}{\partial t}+\boldsymbol{V} \cdot \nabla_{\boldsymbol{r}} f-\nabla_{\boldsymbol{r}} \mathcal{U} \cdot \nabla_{\boldsymbol{V}} f=0$.

This is a consequence of the Hamiltonian flow, which preserves volume, i.e. satisfies the Liouville theorem: the density of any element of phase space remains constant during its motion. Jeans showed that the fundamental equation of stellar dynamics was a particular case of the Boltzmann equation from the kinetic theory of gases,

$\frac{\partial f}{\partial t}+\dot{\boldsymbol{r}} \cdot \nabla_{r} f+\dot{\boldsymbol{V}} \cdot \nabla_{V} f=C(f, f)$,

where the collision term of the right-hand side may be assumed to be null in two cases. First, if the effect of the irregular forces, such as star encounters, is negligible. Second, if the phase density is invariant with respect to the irregular forces, that is, when the number of points leaving any space volume as a result of encounters is balanced by those which enter the volume for the same reason. In both cases, the Liouville theorem is satisfied.

According to Jeans' direct problem, Eq. (2) is a linear and homogeneous partial differential equation for $f$, for a given potential $\mathcal{U}$. An immediate consequence of the Liouville theorem is that if $I_{1}, I_{2}, \ldots, I_{6}$ are any six functional independent integrals of the stellar motion for a given potential, according to Eq. (1), then the phase space density function must be of the form $f(t, \boldsymbol{r}, \boldsymbol{V})=f\left(I_{1}, I_{2}, \ldots, I_{6}\right)$, where the quantity on the righthand side stands for an arbitrary function of the specified arguments, on the condition that the mass of the system be finite and that the density in the phase space be non-negative. The phase space distribution function is itself an integral of motion. Therefore the integrals of motion univocally determine the orbit of any star in the phase space.

\subsection{Isolating integrals}

The phase density, by its physical significance, must be a onevalued function of the six phase coordinates (e.g. Ogorodnikov 1965). If the stellar system is time-independent, the phase space remains decomposed in a set of disjoint hypersurfaces corresponding to different integral values, although if the system is time-dependent, the orbits may intersect for different times. In any case, for a fixed time $t_{0}$, the integrals must define a family of level curves of the phase space. Then, only the isolating integrals, by meaning that they satisfy the condition of being onevalued, can appear as an argument in the phase density. Analytic integrals are always isolating, but non-classic integrals, which are implicit in a numerical integration of an orbit, are usually non-isolating.

When some kinematic knowledge about the stellar system is available, such as that concerning the integrals of motion, if the density function $f$ is already known, Eq. (2) may be alternatively interpreted as a linear, non-homogeneous partial differential equation for $\mathcal{U}$. For example, the velocity distribution of some stellar groups can be assumed, after a transient period, of Maxwell type, Schwarzschild type, or ellipsoidal shaped (e.g. de Zeeuw \& Lynden-Bell 1985). This viewpoint is a functional approach, also known as Jeans' inverse problem, which generally focuses on the study of a single stellar population ${ }^{1}$ (or may be used to define a statistical population), although it could be combined with a mixture model to get a more complete portrait of the velocity distribution (e.g. Cubarsi 1990). With this viewpoint, there is no need of the collisions term. On the contrary, it is assumed that there are sufficient collisions to keep the system in statistical equilibrium, according to the specific phase space density function or the particular integrals of motion. In other

\footnotetext{
1 In such a case, the Newton-Poisson equation, which relates the gravitational potential to the total stellar density, is useless, since unknown stellar populations, including gas and dark matter, do contribute to the gravitational field.
} 
words, it is assumed that the phase space density function is invariant under the collisional operator $C(f, f)$. The idea comes from the original works on statistical dynamics (Chandrasekhar 1943), where the collisional term, accounting for diffusion and frictional processes, is exactly what is needed to conserve the energy of the whole system and leave the Maxwellian distribution invariant. Therefore, it is not surprising that Lynden-Bell (1967), in studying the equilibrium distribution achieved after a violent relaxation process, induced by rapid fluctuations of the gravitational field, obtains a similar smooth distribution function for a rotating elliptical system as Chandrasekhar (1942). Notice, however, that the former uses the statistical dynamics approach, while the latter in this case faces the problem from analytical dynamics. Other examples are described in Ogorodnikov (1965) when deriving the most probable phase distribution after an efficient relaxation mechanism.

If $\mathcal{P}(t, \boldsymbol{r}, \boldsymbol{V})$ is an isolating integral of motion, continuous and differentiable in its arguments, for any fixed time $t_{0}$, the equation $\mathcal{P}\left(t_{0}, \boldsymbol{r}, \boldsymbol{V}\right)=C$ must define a one-parameter family of fivedimensional surfaces filling all the phase space $\Gamma_{r} \times \Gamma_{V}$, for all the possible values of the constant $C \in I_{P}$. If we assume that the phase density can be written as $f(t, \boldsymbol{r}, \boldsymbol{V})=f(\mathcal{P})$, then $f(\mathcal{P})$ is also an isolating integral of motion, which must define, for the same fixed time $t_{0}$, another uniparametric family of curves $f(\mathcal{P})=K \in I_{f(P)}$, associated with the same set of hypersurfaces filling $\Gamma_{r} \times \Gamma_{V}$. To each level curve of the former family corresponds one, and only one, level curve of the later family, so that $K=f(C)$. Thus, we can assume that the inverse $f^{-1}$ is, like $f$, differentiable in the interior of its domain. Hence, the following inequality must be fulfilled,

$\frac{\mathrm{d} f(\mathcal{P})}{\mathrm{d} \mathcal{P}} \neq 0$.

In other words, $f(\mathcal{P})$ is a strictly increasing or decreasing, smooth function of the argument, in any open set within the interval $I_{P}$. This is a basic property used in Sect. 4 for the general solution of the closure problem.

A typical example of this situation is the generalised Schwarzschild distribution, with $\mathcal{P}=Q+\sigma$, where $Q=\boldsymbol{u}^{\mathrm{T}} \cdot \boldsymbol{A}_{2} \cdot \boldsymbol{u}$ is a quadratic, positive definite form depending on the peculiar velocity $\boldsymbol{u}$, where the second-rank symmetric tensor $\boldsymbol{A}_{2}$ and the scalar function $\sigma$ depend only on time and position. Then, owing to Eq. (4), we can express the collisionless Boltzmann equation in either of the following forms

$\frac{\mathrm{D} f(\mathcal{P})}{\mathrm{D} t}=\frac{\mathrm{d} f(\mathcal{P})}{\mathrm{d} \mathcal{P}} \frac{\mathrm{D} \mathcal{P}}{\mathrm{D} t}=0 \Longleftrightarrow \frac{\mathrm{D} \mathcal{P}}{\mathrm{D} t}=0$.

For the generalised Schwarzschild distribution, Chandrasekhar (1942) obtained a system of twenty partial differential equations for $\boldsymbol{A}_{2}, \sigma$, the mean velocity, and the potential, which was equivalent to the collisionless Boltzmann equation.

\subsection{Moment equations}

In the very beginning of the book, Chandrasekhar (1942) outlines the appropriate conditions to define a unique local standard of rest for describing the motions in a given relatively small volume of the Galaxy. The conditions are related to a continuous estimation of the centroid velocity within this volume and to a slow varying distribution function, which could be referred to as regularity conditions. He concludes that the stellar systems can be divided into those for which the notion of local standard of rest (and, by extension, higher velocity moments) is of significance and those for which it is not. Among the latter we can mention the systems dominated by a phase mixing process ${ }^{2}$ (e.g. Binney \& Tremaine 1987), for which a macroscopic, coarsegrained distribution function may be defined in contrast with the true, fine-grained distribution function, although the coarsegrained distribution function of a mixing system would not satisfy the collisionless Boltzmann equation. We shall focus on the first class of stellar systems, for which the mean velocity and similar statistics are meaningful.

The macroscopic properties of a stellar system are usually described from the symmetric tensors of $n$-order moments $\boldsymbol{m}_{n}$. They provide indirect information of the phase space density function $f(t, \boldsymbol{r}, \boldsymbol{V})$ for fixed values of time and position. The current paper follows the notation and definitions of Paper I. Therefore, here we shall only recall that the tensor $\boldsymbol{m}_{n}$ has $\left(\begin{array}{c}n+2 \\ 2\end{array}\right)$ different elements according to the expression $m_{i_{1} i_{2} \ldots i_{n}}=$ $\left\langle V_{i_{1}} V_{i_{2}} \ldots V_{i_{n}}\right\rangle$, whose indices belong to the set $\{1,2,3\}$, depending on the velocity component. Instead of the component notation, namely the Latin indices notation, a notation to make the velocity powers explicit may also be used, the Greek indices notation, according to $m_{\alpha \beta \gamma}=\left\langle V_{1}^{\alpha} V_{2}^{\beta} V_{3}^{\gamma}\right\rangle$. The normalisation of the density function is given by $m_{0}=1$, and the mean velocity, or velocity of the centroid, is given by $\boldsymbol{m}_{1}=\boldsymbol{v}(t, \boldsymbol{r})$. In a similar way, the symmetric tensor of $n$-order central moments $\boldsymbol{\mu}_{n}$ is obtained from the peculiar velocity $\boldsymbol{u}=\boldsymbol{V}-\boldsymbol{m}_{1}$, with elements $\mu_{i_{1} i_{2} \ldots i_{n}}=\left\langle u_{i_{1}} u_{i_{2}} \ldots u_{i_{n}}\right\rangle$. In this case, $\mu_{0}=1$ and $\boldsymbol{\mu}_{1}=\mathbf{0}$.

It is well known that the stellar hydrodynamic equations, usually referred to as the moment equations, are obtained when above kinematic statistics are introduced into the collisionless Boltzmann equation. By multiplying Eq. (3) by the $n$-tensor power of the star velocity, and by integrating over the whole velocity space we get

$\int_{\Gamma_{V}}(\boldsymbol{V})^{n} \frac{\mathrm{D} f}{\mathrm{D} t} \mathrm{~d}^{3} \boldsymbol{V}=(\mathbf{0})^{n} ; \quad n \geq 0$,

where in the integration process the following boundary conditions are assumed because there are no stars with velocity beyond $\Gamma_{V}$

$\boldsymbol{V} \rightarrow \partial \Gamma_{V} \Longrightarrow(\boldsymbol{V})^{n} f(t, \boldsymbol{r}, \boldsymbol{V}) \rightarrow(\boldsymbol{0})^{n}, n \geq 0$.

It is always assumed that the foregoing integrals do exist, as those of the velocity the moments. The moment equations are physically meaningful when they are written in terms of the central moments or pressures, in the reference frame associated with the local centroid (Paper II), so that for each value of $n$, the tensor equation Eq. (6) provides a conservation law along the centroid trajectory. Indeed, the physical interpretation of the Chandrasekhar's (1942) system of equations is more evident from the corresponding set of hydrodynamic equations, in terms of mass conservation, acceleration of the centroid, and transfer of even- and odd-order pressures, as shown in Paper II.

\section{Maximum entropy function}

Although the closure problem will be studied for a wider family of density functions, a functional approach with maximum

\footnotetext{
${ }^{2}$ For the Galaxy and, in general, for stellar systems larger than globular clusters, the forces acting on a star can exclusively be associated with a mean gravitational field, by neglecting the random forces due to stellar encounters. In the solar neighbourhood, assuming that the Galaxy has reached an equilibrium configuration, the potential is usually taken as explicitly time-independent. Then, the Hamiltonian flow possesses the energy integral, is always nonergodic and, therefore, nonmixing (e.g. Arnold \& Avez 1968).
} 
entropy distributions is firstly considered. Basically, they are generalised exponential distributions, including the exponential, normal, lognormal, gamma, and beta as special cases. The main properties of these density functions are detailed in Paper I. From a thermodynamical viewpoint, a maximum entropy density is a function (a) that depends on a linear combination of the collisional invariants, i.e. mass, momentum, and energy; (b) for which the collision term of the Boltzmann equation is exactly zero, i.e., it is a solution of the collisionless Boltzmann equation; and (c) that minimises Boltzmann's entropy functional. This solution represents a local equilibrium state, in the sense that other solutions to the Boltzmann equation become asymptotically close to it. But thermodynamical entropy is a particular case of information entropy. If the conserved macroscopic quantities involve moments accounting for mass and energy, Shannon's information entropy takes its maximum value in the form of Maxwellian distributions. If the conservation extends to all the second moments, the information entropy takes the extreme value for Gaussian distributions. In general, the number of constraints may involve higher-order moments, by reflecting a more complex situation in which the stars interact with the potential and with themselves. Indeed, the moment constraints are a direct consequence of the isolating integrals of the stellar motion, or more precisely, they reflect particular combinations of the integrals of motion which are conserved. Therefore, we can adopt the viewpoint of Jeans' inverse problem.

The form of a maximum entropy function is

$f(t, \boldsymbol{r}, \boldsymbol{V})=\mathrm{e}^{\mathcal{P}(\boldsymbol{V})}$,

where $\mathcal{P}(\boldsymbol{V})$ represents a power series in the velocity components with coefficients depending on time and position. It contains as many terms as the number of available moment constraints, so that each coefficient is related to a single moment constraint.

A maximum entropy distribution has a very simple functional form, allowing us to show straightforwardly the equivalence of the collisionless Boltzmann equation and a finite set of moment equations. Afterwards, it can be easily generalised to non-maximum entropy distributions. We write the phase space density function as

$f=\mathrm{e}^{\mathcal{P}_{n}}, \quad \mathcal{P}_{n}=\sum_{\alpha+\beta+\gamma \leq n} \lambda_{\alpha \beta \gamma}(t, \boldsymbol{r}) V_{1}^{\alpha} V_{2}^{\beta} V_{3}^{\gamma}$,

where the subindex $n$ does not represent the number of terms, but the polynomial degree. If the velocity domain $\Gamma_{V}$ is infinite, the polynomial $\mathcal{P}_{n}$ must be upper bounded to satisfy the integrability conditions given by Eq. (7). Otherwise, as discussed in Paper I, a truncated bell-shaped velocity distribution might be considered.

\subsection{Fundamental system of equations}

The conservation of the integral of motion $\mathcal{P}_{n}$ is expressed by substituting Eq. (9) into Eq. (5), by considering Eq. (4). The equation

$\frac{\mathrm{D} \mathcal{P}_{n}}{\mathrm{D} t}=0$

represents a linear and homogeneous system of partial differential equations for the coefficients of $\mathcal{P}_{n}$. Thus, taking the collisionless form of Eq. (3) into account, the above equation can be explicitly written as

$\frac{\partial \mathcal{P}_{n}}{\partial t}+V_{k} \frac{\partial \mathcal{P}_{n}}{\partial r_{k}}-\frac{\partial \mathcal{U}}{\partial r_{k}} \frac{\partial \mathcal{P}_{n}}{\partial V_{k}}=0$
For the remaining part of this section, it is more convenient to write Eq. (9) by using Latin indices, along with Einstein's summation criterion for repeated indices,

$\mathcal{P}_{n}=\lambda_{0}+\lambda_{i} V_{i}+\lambda_{i j} V_{i} V_{j}+\ldots+\lambda_{i_{1} \ldots i_{n}} V_{i_{1}} \ldots V_{i_{n}}$.

The coefficients $\lambda_{i_{1} \ldots i_{k}}$ in the term corresponding to the $k$ th-power of the velocities are symmetric, so that we have $\left(\begin{array}{c}k+2 \\ 2\end{array}\right)$ different coefficients. Hence, up to the $n$ th-power, it makes $\sum_{k=0}^{n}\left(\begin{array}{c}k+2 \\ k\end{array}\right)=$ $\left(\begin{array}{c}n+3 \\ 3\end{array}\right)$ different coefficients. The correspondence between the Greek and Latin indices notation for the coefficients of $\mathcal{P}_{n}$, depending on the use of either Eq. (9) or (12), is given by

$\lambda_{\alpha \beta \gamma}=\frac{k !}{\alpha ! \beta ! \gamma !} \lambda_{\underbrace{1 \ldots 1}_{\alpha}}^{2 \ldots 2} \underbrace{2 \ldots 3}_{\beta} ; \quad k=\alpha+\beta+\gamma$.

Then, Eq. (11) becomes

$$
\begin{aligned}
\frac{\mathrm{D} \mathcal{P}_{n}}{\mathrm{D} t}= & \frac{\partial \lambda_{0}}{\partial t}+\frac{\partial \lambda_{i}}{\partial t} V_{i}+\frac{\partial \lambda_{i j}}{\partial t} V_{i} V_{j}+\ldots+\frac{\partial \lambda_{i_{1} \ldots i_{n}}}{\partial t} V_{i_{1}} \ldots V_{i_{n}}+\frac{\partial \lambda_{0}}{\partial r_{k}} V_{k} \\
& +\frac{\partial \lambda_{i}}{\partial r_{k}} V_{i} V_{k}+\frac{\partial \lambda_{i j}}{\partial r_{k}} V_{i} V_{j} V_{k}+\ldots+\frac{\partial \lambda_{i_{1} \ldots i_{n}}}{\partial r_{k}} V_{i_{1}} \ldots V_{i_{n}} V_{k} \\
& -\frac{\partial \mathcal{U}}{\partial r_{k}}\left(\lambda_{i} \delta_{i k}+\lambda_{i j} \delta_{i k} V_{j}+\lambda_{i j} \delta_{j k} V_{i}+\ldots+\lambda_{i_{1} \ldots i_{n}} \delta_{i_{1} k} V_{i_{2}} \ldots V_{i_{n}}\right. \\
& \left.+\ldots+\lambda_{i_{1} \ldots i_{n}} \delta_{i_{n} k} V_{i_{1}} \ldots V_{i_{n-1}}\right)=0 .
\end{aligned}
$$

To simplify the notation, by permuting some indices and considering the symmetry of the coefficients, we define the following tensors

$\Lambda_{0}=\frac{\partial \lambda_{0}}{\partial t}-\frac{\partial \mathcal{U}}{\partial r_{i}} \lambda_{i}$

$\Lambda_{i}=\frac{\partial \lambda_{i}}{\partial t}+\frac{\partial \lambda_{0}}{\partial r_{i}}-2 \frac{\partial \mathcal{U}}{\partial r_{k}} \lambda_{i k}$

$\Lambda_{i j}=\frac{\partial \lambda_{i j}}{\partial t}+\frac{\partial \lambda_{i}}{\partial r_{j}}-3 \frac{\partial \mathcal{U}}{\partial r_{k}} \lambda_{i j k}$

$\cdots$

$\Lambda_{i_{1} \ldots i_{n-1}}=\frac{\partial \lambda_{i_{1} i_{2} \ldots i_{n-1}}}{\partial t}+\frac{\partial \lambda_{i_{1} i_{2} \ldots i_{n-2}}}{\partial r_{n-1}}-n \frac{\partial \mathcal{U}}{\partial r_{i_{n}}} \lambda_{i_{1} i_{2} \ldots i_{n}}$,

$\Lambda_{i_{1} \ldots i_{n}}=\frac{\partial \lambda_{i_{1} i_{2} \ldots i_{n}}}{\partial t}+\frac{\partial \lambda_{i_{1} i_{2} \ldots i_{n-1}}}{\partial r_{n}}$

$\Lambda_{i_{1} \ldots i_{n+1}}=\frac{\partial \lambda_{i_{1} i_{2} \ldots i_{n}}}{\partial r_{n+1}}$

which are also symmetric quantities (hence, it suffices to consider only the indices satisfying $\left.1 \leq i_{1} \leq i_{2} \leq \ldots \leq 3\right)$. Therefore, from Eqs. (14) and (15), the collisionless Boltzmann equation can be written in terms of the elements of tensors $\boldsymbol{\Lambda}_{k}$ as $\frac{\mathrm{D} \mathcal{P}_{n}}{\mathrm{D} t}=\Lambda_{0}+\Lambda_{i} V_{i}+\Lambda_{i j} V_{i} V_{j}+\ldots+\Lambda_{i_{1} \ldots i_{n+1}} V_{i_{1}} \ldots V_{i_{n+1}}=0$

which must be fulfilled for all possible values of the velocity. Then, since each symmetric tensor $\boldsymbol{\Lambda}_{k}, k=0, \ldots, n+1$, has $\left(\begin{array}{c}k+2 \\ 2\end{array}\right)$ different elements, a number of $\left(\begin{array}{c}n+4 \\ 3\end{array}\right)$ scalar equations are in total involved. They are

$$
\begin{aligned}
& \Lambda_{0}=0, \\
& \Lambda_{i}=0, \\
& \Lambda_{i j}=0, \\
& \cdots \\
& \Lambda_{i_{1} \ldots i_{n+1}}=0 .
\end{aligned}
$$

In this way, the collisionless Boltzmann equation is equivalent to the above system of partial differential equations, depending on the variables $t$ and $\boldsymbol{r}$. 


\subsection{Closure of moment equations}

We are ready to see that the foregoing system of equations is equivalent to a similar set of independent moment equations, and that the remaining hydrodynamic equations are obtained from linear combinations of them.

Firstly, from Eqs. (6) and (10), we write the general expression of the moment equations for an arbitrary order $l \geq 0$,

$\mathcal{M}_{\alpha \beta \gamma}=\int_{\Gamma_{V}} V_{1}^{\alpha} V_{2}^{\beta} V_{3}^{\gamma} \frac{\mathrm{D} \mathcal{P}_{n}}{\mathrm{D} t} \mathrm{e}^{\mathcal{P}_{n}} \mathrm{~d}^{3} \boldsymbol{V}=0 ; \quad \alpha+\beta+\gamma=l$.

The foregoing relationship can be interpreted as the inner product of $\frac{\mathrm{D} \mathcal{P}_{n}}{\mathrm{D} t}$ and an element $\phi_{\alpha \beta \gamma}=V_{1}^{\alpha} V_{2}^{\beta} V_{3}^{\gamma}$, belonging to the basis of the space of square-integrable functions in $\Gamma_{V}$, with respect to the weight function $\mathrm{e}^{\mathcal{P}_{n}}$. Hence, we can express the first equality in a more compact form,

$\mathcal{M}_{\alpha \beta \gamma}=\left\langle\phi_{\alpha \beta \gamma}, \frac{\mathrm{D} \mathcal{P}_{n}}{\mathrm{D} t}\right\rangle$.

We also write the left-hand side of Eq. (16) by making explicit the velocity powers, in the Greek indices notation, similar to Eq. (9),

$\frac{\mathrm{D} \mathcal{P}_{n}}{\mathrm{D} t}=\sum_{\alpha+\beta+\gamma \leq n+1} \Lambda_{\alpha \beta \gamma}(t, \boldsymbol{r}) V_{1}^{\alpha} V_{2}^{\beta} V_{3}^{\gamma}$.

A relationship similar to Eq. (13) between capital lambdas is hold.

Finally, by substitution of Eq. (20) into (19), we obtain how the moment equations are related to the tensors $\boldsymbol{\Lambda}_{k}$,

$\mathcal{M}_{\alpha \beta \gamma}=\sum_{\iota+\mu+\nu \leq n+1}\left\langle\phi_{\alpha \beta \gamma}, \phi_{\iota \mu \nu}\right\rangle \Lambda_{\iota \mu \nu} ; \quad \alpha+\beta+\gamma=l$.

Notice that the above expression involves the moments $m_{(\alpha+\iota)(\beta+\mu)(\gamma+\nu)}=\left\langle\phi_{\alpha \beta \gamma}, \phi_{\iota \mu \nu}\right\rangle$.

If the foregoing system of equations is restricted to the first set of indices, $0 \leq l \leq n+1$, we get the same number of equations as coefficients $\Lambda_{\mu \nu}$. We are therefore led to a similar situation as in Paper I, where the above system matrix was written as a squared matrix $\boldsymbol{G}_{2}$ of inner products of the velocity components,

$G(\alpha \beta \gamma, \iota \mu \nu)=\left\langle\phi_{\alpha \beta \gamma}, \phi_{\iota \mu \nu}\right\rangle ; \alpha+\beta+\gamma \leq n+1, \iota+\mu+v \leq n+1 .(22)$

Then, $\boldsymbol{G}_{2}$ is a Gram matrix, symmetric, positive definite and, among other well known properties, is invertible. In this notation $^{3}$, we write this subsystem contained in Eq. (21) as

$\mathcal{M}_{\alpha \beta \gamma}=\sum_{\iota+\mu+\nu \leq n+1} G(\alpha \beta \gamma, \iota \mu \nu) \Lambda_{\iota \mu \nu} ; \quad \alpha+\beta+\gamma \leq n+1$.

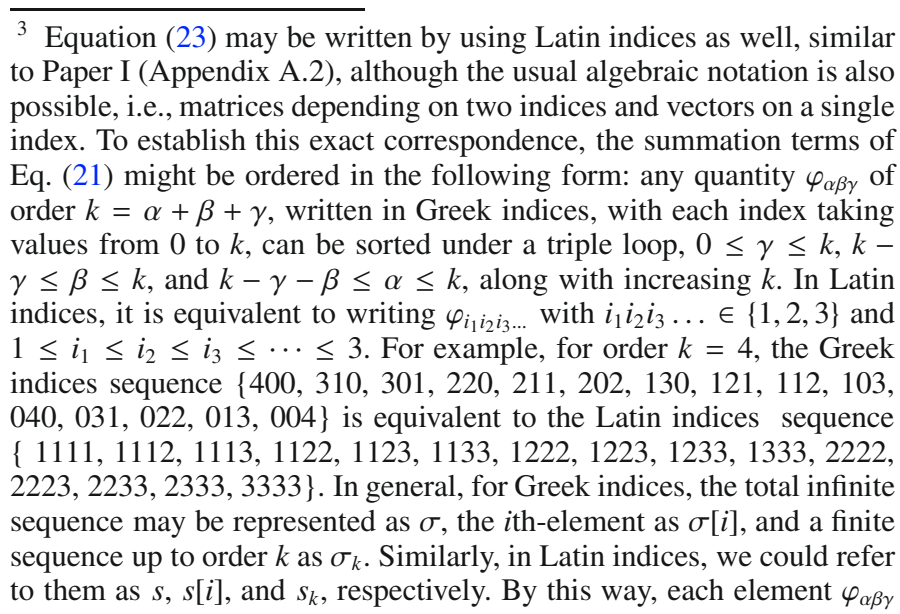

From the above relationship, the first elementary conclusion is that the finite system of equations,

$\Lambda_{\iota \nu}=0 ; \iota+\mu+v \leq n+1$,

corresponding to the collisionless Boltzmann equation, is fulfilled if, and only if, the finite set of hydrodynamic equations,

$\mathcal{M}_{\alpha \beta \gamma}=0 ; \alpha+\beta+\gamma \leq n+1$

is also fulfilled.

A second consequence is that Eq. (23) can be inverted, so that the components $\Lambda_{\mu \nu}$ can be explicitly expressed in terms of linear combinations of the hydrodynamic equations $\mathcal{M}_{\alpha \beta \gamma}$, up to order $n+1$, according to the following relation,

$\Lambda_{\alpha \beta \gamma}=\sum_{\iota+\mu+\nu \leq n+1} G^{-1}(\alpha \beta \gamma, \iota \mu \nu) \mathcal{M}_{\iota \mu \nu} ; \alpha+\beta+\gamma \leq n+1$,

where $G^{-1}(\alpha \beta \gamma, \iota \mu \nu)$ is the corresponding element of the inverse of matrix $\boldsymbol{G}_{2}$. Thus, the finite set of elements $\Lambda_{\alpha \beta \gamma}$, $\alpha+\beta+\gamma \leq n+1$, may be introduced in Eq. (21) to express the infinite hierarchy of higher-order moment equations in terms of the lower-order hydrodynamic equations $\mathcal{M}_{\iota \mu \nu}, \iota+\mu+v \leq n+1$,

$\mathcal{M}_{\alpha \beta \gamma}=\sum_{\alpha^{\prime}+\beta^{\prime}+\gamma^{\prime} \leq n+1}\left\langle\phi_{\alpha \beta \gamma}, \phi_{\alpha^{\prime} \beta^{\prime} \gamma^{\prime}}\right\rangle \sum_{\iota+\mu+\nu \leq n+1} G^{-1}\left(\alpha^{\prime} \beta^{\prime} \gamma^{\prime}, \iota \mu \nu\right) \mathcal{M}_{\iota \mu \nu}$

for $\alpha+\beta+\gamma \geq n+2$.

The foregoing relationship clearly shows the recurrence of the higher-order equations in terms of a finite set of the lowerorder ones. Therefore, for maximum entropy density functions, the closure of the stellar hydrodynamic equations is proven.

\section{Arbitrary function of $\mathcal{P}_{\mathrm{n}}$}

The closure of the moment equations is also valid for any arbitrary density function $f\left(\mathcal{P}_{n}\right)$, even if it is not a maximum entropy function. By following the same steps as in Appendix B of Paper II, it can be shown ${ }^{4}$ that any phase density $f\left(\mathcal{P}_{n}\right)$, which is a square-integrable function with respect to the weight $\mathrm{e}^{\mathcal{P}_{n}}$ in $\Gamma_{V}$, can be expressed as the following convergent power series

$f\left(\mathcal{P}_{n}\right)=\sum_{k=1}^{\infty} \gamma_{k-1} \mathrm{e}^{k \mathcal{P}_{n}}$

could be written as depending on a single index, $\varphi_{\sigma[i]}$, and Eq. (23) would become

$$
\mathcal{M}_{\sigma[l]}=\sum_{\sigma[k] \in \sigma_{n+1}} G_{\sigma[l] \sigma[k]} \Lambda_{\sigma[k]} ; \quad \sigma[l] \in \sigma_{n+1} .
$$

${ }^{4}$ Instead of repeating the full derivation for the above property, the velocity distribution function may be written in similar terms as in Paper II, where it was shown that any arbitrary quadratic density function $f(Q+\sigma)$ could be expressed as a convergent series of the Gaussian functions $\mathrm{e}^{-\frac{1}{2}(Q+\sigma) k}$ with $k \geq 1$. Let us assume an infinite velocity domain $\Gamma_{V}$. Since $\mathcal{P}_{n}$ is upper bounded, there exist a value $\zeta$, which may depend on time and position, such that $\mathcal{P}_{n}<\zeta$ for all velocity $V \in \Gamma_{V}$. Thus, we can write $\mathcal{P}_{n}=-\frac{1}{2}\left(Q_{n}+\sigma\right)$ with $Q_{n}=-2\left(\mathcal{P}_{n}-\zeta\right)$ a positive definite form, and $\sigma=-2 \zeta$. Hence, we are in the appropriate conditions to show that any function $f\left(Q_{n}+\sigma\right)$ can be expressed as a convergent power series in terms of $\mathrm{e}^{-\frac{1}{2}\left(Q_{n}+\sigma\right)}$. The case of a finite velocity domain is quite similar but with the corresponding changes concerning the domain of the variables. Thus, if $\zeta_{1}<\mathcal{P}_{n}<\zeta_{2}$, then the variable $\tau=\frac{1}{2}\left(Q_{n}+\sigma\right)$, defined in Paper II, belongs to the interval $I=\left(-\zeta_{2},-\zeta_{1}\right)$, and the variable $\eta=\mathrm{e}^{-\tau}$ belongs to the interval $J=\left(\mathrm{e}^{-\zeta_{2}}, \mathrm{e}^{-\zeta_{1}}\right)$. 
where the coefficients $\gamma_{k-1}$ are constant, and $f$ depends on time, space, and velocity through $\mathcal{P}_{n}$, as in Eq. (9). If we assume that the distribution function is continuously differentiable in the interior of $\Gamma_{V}$, this generalised Fourier series can be integrated or differentiated term by term without loosing its convergence property.

On the other hand, due to the boundary conditions, Eq. (7), when the velocity $\boldsymbol{V}$ approaches the boundary domain $\Gamma_{V}$, then $\mathcal{P}_{n} \rightarrow-\infty$ and $f\left(\mathcal{P}_{n}\right) \rightarrow 0$. According to Eq. (4), since $f\left(\mathcal{P}_{n}\right)>0, f$ must be an increasing function of $\mathcal{P}_{n}$, so that

$\frac{\mathrm{d} f\left(\mathcal{P}_{n}\right)}{\mathrm{d} \mathcal{P}_{n}}>0$

in the interior of the domain $\Gamma_{r} \times \Gamma_{V}$, for any fixed time.

\subsection{Moment equations}

By taking derivatives in Eq. (28),

$\frac{\mathrm{d} f\left(\mathcal{P}_{n}\right)}{\mathrm{d} \mathcal{P}_{n}}=\sum_{k=1}^{\infty} \gamma_{k-1} k \mathrm{e}^{k \mathcal{P}_{n}}$,

and bearing in mind Eq. (5), we have

$\frac{\mathrm{D} f\left(\mathcal{P}_{n}\right)}{\mathrm{D} t}=\sum_{k=1}^{\infty} \gamma_{k-1} \mathrm{e}^{k \mathcal{P}_{n}} \frac{\mathrm{D}\left(k \mathcal{P}_{n}\right)}{\mathrm{D} t}=0$.

Then, for any $k \neq 0$, Eqs. (12) and (16) are also fulfilled with $\widetilde{\mathcal{P}}_{n}=k \mathcal{P}_{n}, \tilde{\boldsymbol{\lambda}}_{i}=k \boldsymbol{\lambda}_{i}$, and $\widetilde{\boldsymbol{\Lambda}}_{i}=k \boldsymbol{\Lambda}_{i}$; since $\mathcal{P}_{n}$ is linearly dependent on the tensor elements $\lambda_{i}$, and $\frac{\mathrm{D} \mathcal{P}_{n}}{\mathrm{D} t}$ and $\boldsymbol{\Lambda}_{i}$ are similarly related. Then, each term of the above series satisfies the collisionless Boltzmann equation, so that a linear relationship, similar to Eq. (23), holds. Therefore, for each term of the series Eq. (31), we get the integrals

$\mathcal{M}_{\alpha \beta \gamma}^{(k)}=\int_{\Gamma_{V}} \phi_{\alpha \beta \gamma} \frac{\mathrm{D}\left(k \mathcal{P}_{n}\right)}{\mathrm{D} t} \mathrm{e}^{k \mathcal{P}_{n}} \mathrm{~d}^{3} \boldsymbol{V}=0 ; \quad \alpha+\beta+\gamma=l$,

and we sum up, according to the coefficients of the series, so that we obtain

$$
\sum_{k=1}^{\infty} \gamma_{k-1} \mathcal{M}_{\alpha \beta \gamma}^{(k)}=\int_{\Gamma_{V}} \phi_{\alpha \beta \gamma} \sum_{k=1}^{\infty} \gamma_{k-1} \frac{\mathrm{D}\left(k \mathcal{P}_{n}\right)}{\mathrm{D} t} \mathrm{e}^{k \mathcal{P}_{n}} \mathrm{~d}^{3} \boldsymbol{V} .
$$

Hence, according to Eq. (31), we are led to the general expression of the moment equations, which generalises Eq. (18)

$\mathcal{M}_{\alpha \beta \gamma}=\int_{\Gamma_{V}} \phi_{\alpha \beta \gamma} \frac{\mathrm{D} f\left(\mathcal{P}_{n}\right)}{\mathrm{D} t} \mathrm{~d}^{3} \boldsymbol{V}=0$.

For any order $l=\alpha+\beta+\gamma$, the $l$-order moment equation is obtained as linear combination of the moment equations associated with each term of the series Eq. (31),

$\mathcal{M}_{\alpha \beta \gamma}=\sum_{k=1}^{\infty} \gamma_{k-1} \mathcal{M}_{\alpha \beta \gamma}^{(k)}$.

\subsection{Equivalence}

The relationship between the hydrodynamic equations and the collisionless Boltzmann equation is now established by substitution of Eq. (20) in (33),

$\mathcal{M}_{\alpha \beta \gamma}=\int_{\Gamma_{V}} \phi_{\alpha \beta \gamma} \sum_{k=1}^{\infty} \gamma_{k-1}\left(\sum_{\iota+\mu+\nu \leq n+1} \Lambda_{\iota \mu \nu} \phi_{\iota \mu \nu}\right) k \mathrm{e}^{k \mathcal{P}_{n}} \mathrm{~d}^{3} \boldsymbol{V}$.
By reordering terms,

$$
\mathcal{M}_{\alpha \beta \gamma}=\sum_{\iota+\mu+v \leq n+1} \Lambda_{\mu \nu}\left[\int_{\Gamma_{V}} \phi_{\alpha \beta \gamma} \phi_{\iota \mu \nu}\left(\sum_{k=1}^{\infty} \gamma_{k-1} k \mathrm{e}^{k \mathcal{P}_{n}}\right) \mathrm{d}^{3} \boldsymbol{V}\right]
$$

and bearing in mind Eq. (30), we may also write

$$
\mathcal{M}_{\alpha \beta \gamma}=\sum_{\iota+\mu+\nu \leq n+1}\left[\int_{\Gamma_{V}} \phi_{\alpha \beta \gamma} \phi_{\iota \mu \nu} \frac{\mathrm{d} f\left(\mathcal{P}_{n}\right)}{\mathrm{d} \mathcal{P}_{n}} \mathrm{~d}^{3} \boldsymbol{V}\right] \Lambda_{\iota \mu \nu}
$$

Therefore, if only orders $\alpha+\beta+\gamma \leq n+1$ are considered, we are led to a similar relationship as Eq. (21), but with the inner product calculated with the weight function given by Eq. (29), which will be notated with a Gram matrix $\widetilde{\boldsymbol{G}}_{2}$. The resulting integrals are some generalised velocity moments, so that when $f\left(\mathcal{P}_{n}\right)$ is a maximum entropy function then $f\left(\mathcal{P}_{n}\right)=\frac{\mathrm{d} f\left(\mathcal{P}_{n}\right)}{\mathrm{d} \mathcal{P}_{n}}$ and the generalised moments become ordinary velocity moments. Thus we write

$\mathcal{M}_{\alpha \beta \gamma}=\sum_{\iota+\mu+v \leq n+1} \widetilde{G}(\alpha \beta \gamma, \iota \mu \nu) \Lambda_{\iota \mu \nu} ; \alpha+\beta+\gamma \leq n+1$.

The relationship between a finite set of hydrodynamic equations and the collisionless Boltzmann equation, given by Eqs. (23)(25), is now expressed as $\boldsymbol{M}_{n+1}=\widetilde{\boldsymbol{G}}_{2} \boldsymbol{\Lambda}_{n+1}$, so that $\boldsymbol{\Lambda}_{n+1}=$ $\mathbf{0}_{n+1} \Longleftrightarrow \boldsymbol{M}_{n+1}=\mathbf{0}_{n+1}$.

Therefore, for any density function depending on an $n$ degree polynomial function $\mathcal{P}_{n}$, there is a finite set of independent moment equations, for the orders $i=0,1, \ldots, n+1$, which is equivalent to the collisionless Boltzmann equation. Furthermore, a recurrence law for moment equations similar to Eq. (27), but with the weight function given by Eq. (29), is satisfied.

Similar to Paper I, but for the general case of a nonmaximum entropy function, it is possible to prove the linear relationship between the coefficients of the polynomial function $\mathcal{P}_{n}$ and a finite set of extended velocity moments (see Appendix A). Also, as commented in Paper I, for a given density function, the quantities $\lambda_{k}, k \leq n$, are univocally related to the minimum set of moments $\boldsymbol{m}_{k}, k \leq n$, so that all the higher-order moments could be computed from the former set. However, the proven linear relationship makes use of an extended set of moments up to an order $2(n-1)$. Only for $n=2$, the minimum and the extended set of moments match up. For this case, the moment recurrence was explicitly used in Paper II to derivate the redundancy of the higher-order moment equations. However, for $n>2$, the existence of a general analytical relationship involving only the minimum set of moments should be further investigated.

\section{Concluding remarks}

The description of how the Galaxy relaxes towards a steady state is still a matter of debate, but there are two processes that likely play an important role: phase mixing and violent relaxation. Lynden-Bell (1967), in a seminal work, gave a statistical description of how a rapid fluctuating gravitational field produces a relaxation mechanism under the collisionless Boltzmann equation, which involves phase mixing, by changing the coarse-grained phase-space density near the phase point of each star, and violent relaxation, analogous to collisions in a gas, by changing the energy per unit mass of a star. Lynden-Bell's approach leads, for a non-degenerate stellar system, to a Maxwell-Boltzmann macroscopic distribution. Improvements to the previous approach (e.g. Chavanis et al. 1996; Chavanis 1998) take into account, among 
other aspects, the self confinement of the Galaxy, which is related to the incomplete relaxation problem due to the hypothesis of ergodicity; the maximum-entropy production principle to obtain a closure of the relaxation equation of diffusion type (non collisionless) for the coarse-grained distribution function; and the estimation of the diffusion current, which generalises the Chandrasekhar (1943) and Lynden-Bell (1967) equations. These and similar approaches, from a statistical viewpoint, and following the Jeans' direct problem, lead to the most probable distribution function for an equilibrium configuration of the Galaxy, and provide information about the functional form of the distribution function, or about the conserved quantities along the stellar motion, by leading to a distribution function that may take the form $f\left(\mathcal{P}_{n}\right)$.

In this stage, once the system has achieved relaxation, and according to Jeans' inverse problem, the situation can be reversed. It can be approached not from the statistical dynamics viewpoint but from analytical dynamics, by assuming the regularity conditions about the definition of the local standard of rest, the continuity and differentiability of its velocity, and the existence of higher-order velocity moments. We then ask under which circumstances the collisionless Boltzmann equation admits a solution of the form $f\left(\mathcal{P}_{n}\right)$. This is indeed the appropriate context to study the motion of the centroid and the admissible form of the potential function. Therefore, dissipative forces are not considered in the collisionless Boltzmann equation, but are indirectly connected with the functional form of the distribution function. This situation can be also tackled by using the stellar hydrodynamic equations, which explicitly involves the velocity moments. Then, the closure problem necessarily arises of how the infinite hierarchy of moment equations is related to the finite character of the collisionless Boltzmann equation.

In this context, the equivalence of Boltzmann and moment equations had already been investigated for $n=2$. In this case, Eq. (17) corresponds to the Chandrasekhar's (1942) system of equations, which allows us to obtain, under a time-dependent model and different symmetry hypotheses, a quite general solution for the collisionless Boltzmann equation, with the possibility of describing a stellar system with arbitrary mean velocity and orientation of velocity ellipsoid (Sanz-Subirana \& Català-Poch 1987; Sala 1990; Juan-Zornoza \& Sanz-Subirana 1991; Juan-Zornoza 1995). In this case, de Orús (1952) proved that if the Chandrasekhar's equations are fulfilled, the continuity equation and the Jean's equation are also satisfied. In the same vein, working from velocity moments up to fourth-order, JuanZornoza (1995) showed that Chandrasekhar's equations could be derived from the first four hydrodynamic equations. A more general result, for generalised Schwarzschild distributions, was derived in Paper II: the first four hydrodynamic equations, along with a moment recurrence relationship acting as closure condition, make the infinite hierarchy of hydrodynamic equations equivalent to the collisionless Boltzmann equation. Now, the above results have been generalised to any velocity distribution function depending on a polynomial function in the velocity variables.

The degree of this polynomial function, which is an isolating integral of the stellar motion, may be used to quantify the complexity of the velocity distribution. In Paper I, under a maximum entropy approach, this complexity was in some way measured in terms of the necessary set of velocity moments, for obtaining a good fit of the velocity distribution. In the most basic situations, the stellar systems can be approximated by an ellipsoidal distribution, such as for the thin disk, thick disk, or halo, as independent Galactic components (Cubarsi \& Alcobé 2004;
Alcobé \& Cubarsi 2005; Cubarsi et al. 2010). Those systems are well described with moments up to second order and, therefore, according to our results, they can be modelled by using the moment equations up to third order, or the system of Chandrasekhar equations, equivalent to the collisionless Boltzmann equation. Other stellar systems have a slight deviation from the ellipsoidal hypothesis, such as the whole Galactic disc. In Paper I, two samples, namely Sample I from the HIPPARCOS catalogue (ESA 1997) and Sample II from the Geneva-Copenhagen survey (GCS) catalogue (Nordström et al. 2004), were drawn to be representative of the local Galactic disc. The detailed analysis is found in the application section of Paper I. For them, a good fit of the velocity distribution was obtained for a fourth-degree polynomial function. Then, the modelling of the Galactic disc would require accounting for the hydrodynamic equations up to order five, or the equivalent set of equations in Eq. (17). On the other hand, to study large enough stellar groups conforming disc substructures, like those obtained in Paper I from finite velocity domains, i.e., Sample III from the HIPPARCOS catalogue and Sample IV from the GCS catalogue, or such as the samples drawn from limited stellar eccentricity, in particular, for planar eccentricities lower than 0.15 with a local standard of rest velocity of $(-9.72,-11.45,-6.65) \mathrm{km} \mathrm{s}^{-1}$ referred to the Sun, which gave a satisfactory representation of the dominant local moving groups, it was then necessary to approach the velocity distribution with a sixth-degree polynomial function. For these more complex systems, the modelling would require at least moment equations up to the seventh-order.

As a conclusion, we summarise the main results of the paper. The following statements are equivalent:

(a) The velocity distribution depends on an integral of motion which is a polynomial function of degree $n$.

(b) There is an independent set of velocity moments, up to an order $n$, so that the higher-order moments can be expressed in terms of the independent moments.

(c) The collisionless Boltzmann equation is given by a set of differential equations expressed from symmetric tensors of rank up to $n+1$.

(d) The independent moment equations are those of an order of up to $n+1$.

(e) The hydrodynamic equations of an order higher than $n+1$ are redundant.

Acknowledgements. The author thanks the anonymous referee very much for the comments that helped to clarify the appropriate context of the results.

\section{Appendix A}

As in Paper I (Appendix A.1), but now for the general case of an arbitrary density function $f\left(\mathscr{P}_{n}\right)$, we compute the coefficients $\lambda_{k}$, $1 \leq k \leq n$, of Eq. (12), in terms of an extended set of moment constraints, by integrating

$\int_{\Gamma_{V}} \nabla_{\boldsymbol{V}}\left[(\boldsymbol{V})^{m} f\left(\mathcal{P}_{n}\right)\right] \mathrm{d}^{3} \boldsymbol{V}=(\mathbf{0})^{n+1}$

as a result of applying in the integration process the boundary conditions given by Eq. (7). The resulting Gramian system matrix $\widetilde{\boldsymbol{G}}_{2}$ is now a matrix of inner products associated with the basis $\phi_{\alpha \beta \gamma}=V_{1}^{\alpha} V_{2}^{\beta} V_{3}^{\gamma}$, with regard to the weight given by Eq. (29). 
We write the integrand of Eq. (40) in Greek indices, according to Eq. (9), and we assume $\alpha+\beta+\gamma=m, 0 \leq m \leq n-1$.

By taking the $V_{1}$-derivative, we have

$$
\begin{aligned}
\frac{\partial\left(V_{1}^{\alpha} V_{2}^{\beta} V_{3}^{\gamma} f\left(\mathcal{P}_{n}\right)\right)}{\partial V_{1}}= & \alpha V_{1}^{\alpha-1} V_{2}^{\beta} V_{3}^{\gamma} f\left(\mathcal{P}_{n}\right)+V_{1}^{\alpha} V_{2}^{\beta} V_{3}^{\gamma} \frac{\partial \mathcal{P}_{n}}{\partial V_{1}} \frac{\mathrm{d} f\left(\mathcal{P}_{n}\right)}{\mathrm{d} \mathcal{P}_{n}} \\
= & \alpha V_{1}^{\alpha-1} V_{2}^{\beta} V_{3}^{\gamma} f\left(\mathcal{P}_{n}\right) \\
& +V_{1}^{\alpha} V_{2}^{\beta} V_{3}^{\gamma} \sum_{\iota+\mu+v \leq n} \lambda_{\iota \mu \nu} \iota V_{1}^{\iota-1} V_{2}^{\mu} V_{3}^{v} \frac{\mathrm{d} f\left(\mathcal{P}_{n}\right)}{\mathrm{d} \mathcal{P}_{n}}
\end{aligned}
$$

The last summation can be carried out from $\iota \geq 1$ instead of $\iota \geq 0$. Thus, by noting $\iota-1$ as $\iota$, we then have

$$
\begin{aligned}
\frac{\partial\left(V_{1}^{\alpha} V_{2}^{\beta} V_{3}^{\gamma} f\left(\mathcal{P}_{n}\right)\right)}{\partial V_{1}} & =\alpha V_{1}^{\alpha-1} V_{2}^{\beta} V_{3}^{\gamma} f\left(\mathcal{P}_{n}\right) \\
& +V_{1}^{\alpha} V_{2}^{\beta} V_{3}^{\gamma} \sum_{\iota+\mu+\nu \leq n-1} \lambda_{(\iota+1) \mu v}(\iota+1) V_{1}^{\iota} V_{2}^{\mu} V_{3}^{\nu} \frac{\mathrm{d} f\left(\mathcal{P}_{n}\right)}{\mathrm{d} \mathcal{P}_{n}}
\end{aligned}
$$

Similarly, the other derivatives are

$$
\begin{aligned}
\frac{\partial\left(V_{1}^{\alpha} V_{2}^{\beta} V_{3}^{\gamma} f\left(\mathcal{P}_{n}\right)\right)}{\partial V_{2}} & =\beta V_{1}^{\alpha} V_{2}^{\beta-1} V_{3}^{\gamma} f\left(\mathcal{P}_{n}\right) \\
+V_{1}^{\alpha} V_{2}^{\beta} V_{3}^{\gamma} & \sum_{\iota+\mu+\nu \leq n-1} \lambda_{\iota(\mu+1) v}(\mu+1) V_{1}^{\iota} V_{2}^{\mu} V_{3}^{\nu} \frac{\mathrm{d} f\left(\mathcal{P}_{n}\right)}{\mathrm{d} \mathcal{P}_{n}} \\
\frac{\partial\left(V_{1}^{\alpha} V_{2}^{\beta} V_{3}^{\gamma} f\left(\mathcal{P}_{n}\right)\right)}{\partial V_{3}} & =\gamma V_{1}^{\alpha} V_{2}^{\beta} V_{3}^{\gamma-1} f\left(\mathcal{P}_{n}\right) \\
+V_{1}^{\alpha} V_{2}^{\beta} V_{3}^{\gamma} & \sum_{\iota+\mu+\nu \leq n-1} \lambda_{\iota \mu(\nu+1)}(v+1) V_{1}^{\iota} V_{2}^{\mu} V_{3}^{\nu} \frac{\mathrm{d} f\left(\mathcal{P}_{n}\right)}{\mathrm{d} \mathcal{P}_{n}}
\end{aligned}
$$

Then, if the above expressions are substituted into Eq. (40), by using the notation of Eq. (39), we get

$$
\begin{aligned}
& -\alpha m_{(\alpha-1) \beta \gamma}=\sum_{\iota+\mu+v \leq n-1} \widetilde{G}(\alpha \beta \gamma, \iota \mu \nu)(\iota+1) \lambda_{(\iota+1) \mu v}, \\
& -\beta m_{\alpha(\beta-1) \gamma}=\sum_{\iota+\mu+v \leq n-1} \widetilde{G}(\alpha \beta \gamma, \iota \mu \nu)(\mu+1) \lambda_{\iota(\mu+1) v}, \\
& -\gamma m_{\alpha \beta(\gamma-1)}=\sum_{\iota+\mu+v \leq n-1} \widetilde{G}(\alpha \beta \gamma, \iota \mu \nu)(v+1) \lambda_{\iota \mu(v+1)} .
\end{aligned}
$$

According to this notation, all the moments with a negative index must be considered null. The elements of tensors $\lambda_{k}, 1 \leq k \leq n$, involved in Eq. (9) ( $\lambda_{0}$ is the normalisation factor), are explicitly obtained in terms of the generalised moments up to an order $2(n-1)$, as well as of the ordinary velocity moments up to an order $n-2$, by inverting the above system of equations:

$$
\begin{array}{ll}
\lambda_{(\alpha+1) \beta \gamma}=\frac{-1}{\alpha+1} \sum_{\iota+\mu+\nu \leq n-1} & \widetilde{G}^{-1}(\alpha \beta \gamma, \iota \mu \nu) \iota m_{(\iota-1) \mu \nu}, \\
\lambda_{\alpha(\beta+1) \gamma}=\frac{-1}{\beta+1} \sum_{\iota+\mu+v \leq n-1} \widetilde{G}^{-1}(\alpha \beta \gamma, \iota \mu \nu) \mu m_{\iota(\mu-1) v}, \\
\lambda_{\alpha \beta(\gamma+1)}=\frac{-1}{\gamma+1} \sum_{\iota+\mu+\nu \leq n-1} \widetilde{G}^{-1}(\alpha \beta \gamma, \iota \mu \nu) v m_{\iota \mu(\nu-1)} .
\end{array}
$$

The foregoing expressions are valid for $\alpha+\beta+\gamma \leq n-1$, and $\widetilde{G}^{-1}(\alpha \beta \gamma, \iota \mu \nu)$ is the corresponding element of the inverse of the matrix $\widetilde{\boldsymbol{G}}_{2}$.

Once the polynomial coefficients are calculated, it is possible to express the higher-order generalised moments in terms of them, making use of Eq. (43), for $\alpha+\beta+\gamma \geq n$, by using the corresponding inner products $\left\langle\phi_{\alpha \beta \gamma}, \phi_{\iota \mu \nu}\right\rangle$ with the new weight, instead of $\widetilde{G}(\alpha \beta \gamma, \iota \mu \nu)$.

\section{References}

Alcobé, S., \& Cubarsi, R. 2005, A\&A, 442, 929

Arnold, V. I., \& Avez, A. 1968, Ergodic Problems of Classical Mechanics (New York: W.A. Benjamin Inc.)

Binney, J., \& Tremaine, S. 1987, Galactic Dynamics (Princeton: Princeton University Press)

Chandrasekhar, S. 1942, Principles of Stellar Dynamics (New York: Dover Publications Inc.)

Chandrasekhar, S. 1943, NYASA, 45, 131 (included in later versions of the book Principles of Stellar Dynamics)

Chavanis, P.-H. 1998, MNRAS, 300, 981

Chavanis, P.-H., Sommeria, J., \& Robert, R. 1996, ApJ, 471, 385

Cubarsi, R. 1990, AJ, 99, 1558

Cubarsi, R. 2007, MNRAS, 207, 380

Cubarsi, R. 2010, A\&A, 510, A103

Cubarsi, R., \& Alcobé, S. 2004, A\&A, 427, 131

Cubarsi, R., Alcobé, S., Vidojević, S., \& Ninković, S. 2010, A\&A, 510, A102

Cuddeford, P., \& Amendt, P. 1991, MNRAS, 253, 427

de Orús, J. J. 1952, Ph.D. Thesis, Collectanea Mathematica, V(2), University of Barcelona, Barcelona

de Zeeuw, P. T., \& Lynden-Bell, D. 1985, MNRAS, 215, 713

ESA 1997, The Hipparcos Catalogue, ESA SP-1200

Hénon, M. 1982, A\&A, 114, 211

Jarvis, B. J., \& Freeman, K. C. 1985, ApJ, 295, 324

Juan-Zornoza, J. M. 1995, Ph.D. Thesis (ISBN: 84-475-0767-X), University of Barcelona, Barcelona

Juan-Zornoza, J. M., \& Sanz-Subirana, J. 1991, Ap\&SS, 185, 95

Lynden-Bell, D. 1967, MNRAS, 136, 101

Nordström, B., Mayor, M., Andersen, J., et al. 2004, A\&A, 418, 989

Ogorodnikov, K. F. 1965, Dynamics of Stellar Systems (Oxford: Pergamon Press)

Oort, J. H. 1965, in Galactic Structure, ed. A. Blaauw, \& M. Schmidt (Chicago: University of Chicago Press)

Sala, F. 1990, A\&A, 235, 85

Sanz-Subirana, J., \& Català-Poch M. A. 1987, in 10th ERAM of the IAU, 4, 267

van der Marel, R. P. 1991, MNRAS, 248, 515

Vandervoort, P. O. 1975, ApJ, 195, 333 